\title{
Antioxidant Property Enhancement of Sweet Potato Flour under Simulated Gastrointestinal pH
}

\author{
Kim Wei Chan ${ }^{1, *}$, Nicholas M. H. Khong ${ }^{1}$, Shahid Iqbal ${ }^{2}$, Imam Mustapha Umar ${ }^{1}$ and \\ Maznah Ismail ${ }^{1}$ \\ 1 Laboratory of Molecular Biomedicine, Institute of Bioscience, Universiti Putra Malaysia, \\ 43400 UPM Serdang, Selangor , Malaysia; E-Mails: nmhkhong@gmail.com (N.M.H.K.); \\ mustyimam@hotmail.com (I.M.U.); maznah@medic.upm.edu.my (M.I.) \\ 2 Department of Chemistry, University of Sargodha, Sargodha 40100, Pakistan; \\ E-Mail: ranashahid313@gmail.com
}

* Author to whom correspondence should be addressed; E-Mail: chankw@ibs.upm.edu.my or chankw_antioxidant@yahoo.com; Tel.:+60-3-8947-2145; Fax:+60-3-8947-2116.

Received: 30 May 2012; in revised form: 4 July 2012 / Accepted: 5 July 2012 /

Published: 19 July 2012

\begin{abstract}
Sweet potato is known to be rich in healthful antioxidants, but the stability of its antioxidant properties under gastrointestinal $\mathrm{pH}$ is very much unknown. Hence, this study aimed to evaluate the changes in antioxidant properties (total contents of phenolics and flavonoids as well as antioxidant activity) of sweet potato flour (SPF) under simulated gastrointestinal $\mathrm{pH}$ conditions. It was found that the yield of SPF crude phenolic extract increased from 0.29 to $3.22 \mathrm{~g} / 100 \mathrm{~g}$ SPF upon subjection to gastrointestinal $\mathrm{pH}$ conditions $(p<0.05)$. Also elevated significantly were the total phenolic content (TPC), total flavonoid content (TFC) and antioxidant activity of SPF $(p<0.05)$. In summary, the antioxidant properties of SPF were enhanced under gastrointestinal $\mathrm{pH}$ conditions, suggesting that SPF might possess a considerable amount of bound phenolic and other antioxidative compounds. The antioxidant properties of SPF are largely influenced by $\mathrm{pH}$ and thus might be enhanced during the in vivo digestive process.
\end{abstract}

Keywords: sweet potato flour; antioxidant activity; phenolic content; flavonoid content; simulated gastrointestinal $\mathrm{pH}$ 


\section{Introduction}

Oxidative stress, which is defined as the imbalance between pro-oxidants and antioxidants produced in biological systems, pose huge implications in the process of aging and development of chronic diseases such as arteriosclerosis, cancers and diabetes. Reactive oxygen and nitrogen species formed in excess in any biological system destroy cellular components such as lipids, proteins and deoxyribonucleic acid (DNA) or cause a decrease in the capability of antioxidant defenses [1]. Aside from the normal bodily processes that result in the formation of reactive species, dietary, lifestyle and environmental factors, e.g., smoking, alcohol consumption and infections could accelerate the generation of harmful free radicals [2]. Therefore, antioxidants are needed to not only reduce the level of oxidative stress common in many chronic diseases, but also to serve as adjuvants to other standard therapies in order to provide a synergistic effect in combating chronic diseases [2,3]. Antioxidants could be augmented by dietary supplementation with natural antioxidants preferably from routine dietary items [4-6]. Except for the synergists, antioxidants could be classified into either primary or secondary antioxidants [7]. Primary antioxidants or chain-breaking antioxidants are those which actively inhibit oxidation reactions while secondary antioxidants or preventive antioxidants refer to those which inhibit or slow down oxidation indirectly through mechanisms of iron chelation, quenching of triplet oxygen, etc. In this case, phenolic compounds are considered unique as they can exhibit both the properties of a primary antioxidant [7] as well as a secondary antioxidant [8].

Sweet potato (SP) is cultivated extensively for its nutritious value across many regions of the world. It is commonly consumed by a significant number of people as a source of staple food. Sweet potato (SP) is specifically known to contain significant amounts of vitamins and minerals, and is reported to possess rich antioxidant contents especially in the form of phenolics [9-11]. Although SP may contain large amounts of phenolics, not all is biologically accessible. The challenge actuality lies in understanding what these phenolics have to undergo in the digestive tract by humans and what processes are involved until absorbed. Structural modifications as well as alterations in functional properties caused mainly by $\mathrm{pH}$ changes along different parts of the gastrointestinal tract could interfere with phenolic actions and, thus, affect bioefficacy. Many studies have pointed out significant influences of gut conditions on the bioaccessibility of phytochemicals [12,13]. However, results of studies on the effects of $\mathrm{pH}$ changes on phenolic compounds have not been consistent. Specifically, Vallejo et al. [14] and Perez-Vincente [15] reported degradation of phenolics upon subjection to gastrointestinal conditions while D'Archivio et al. [13] disagreed with such a concept by suggesting that the phenolics bound to food matrices are usually released under gut conditions which increases their availability for absorption and hence action. Huang et al. [16] described that antioxidants such as Trolox and $\alpha$-tocopherol become more stable under gut $\mathrm{pH}$ conditions, hence the ability to retain their activity. We hypothesized that the phenolics and flavonoids of SP are not lost when subjected to simulated gastrointestinal conditions and thus, the present study aimed to investigate such phenomena by simulating gastrointestinal $\mathrm{pH}$ changes in vitro. The present study also sought to evaluate the effects of gastrointestinal $\mathrm{pH}$ conditions on the antioxidant activity of sweet potato flour (SPF). No previous report describing the alteration of phenolics, flavonoids and antioxidant activities upon subjection to simulated gastrointestinal $\mathrm{pH}$ conditions have been presented. Findings from this study will lead to 
better understanding of SPF phenolics upon ingestion and how amenable they are for absorption and other beneficial effects on the body.

\section{Results and Discussion}

\subsection{Extract Yield, Total Phenolic Content (TPC) and Total Flavonoid Content (TFC)}

The yield of crude phenolic extracts of treated (SPF subjected to gastrointestinal $\mathrm{pH}$ conditions) and non-treated (control) sweet potato flour (SPF) is presented in Table 1. It is evident that treatment of SPF under simulated gastrointestinal $\mathrm{pH}$ elevated the yield of crude phenolic extract significantly $(p<0.05)$. The yield of crude phenolics extract was approximately 11 fold higher upon subjection of SPF to simulated gastrointestinal conditions. As the protective effects of cereal fibers depend on their solubility, the increased extract yield of treated SPF is indicative of improved digestibility of SPF upon ingestion.

Table 1. Extract yield of sweet potato flour (SPF) as subjected to simulated gastrointestinal $\mathrm{pH}$ conditions.

\begin{tabular}{cc}
\hline Sweet Potato Flour (SPF) & Extract Yield (g/100g SPF) \\
\hline Non-treated & $0.29 \pm 0.05^{\mathrm{a}}$ \\
Treated & $3.22 \pm 0.65^{\mathrm{b}}$ \\
\hline
\end{tabular}

${ }^{\mathrm{a}, \mathrm{b}}$ : Results obtained from means of three determinations \pm standard deviation. Different letters within the same column indicate significant difference $(p<0.05)$.

Table 2 shows the total phenolic (TPC) and total flavonoid (TFC) contents of treated and non-treated SPF. Generally, TPC and TFC of SPF were all found to be significantly increased $(p<0.05)$ under gastrointestinal $\mathrm{pH}$ conditions. TPC of SPF under gastrointestinal $\mathrm{pH}$ conditions was found to be elevated two-fold, whereas noticeably, TFC was raised 10 fold. The significant increase in TPC of SPF following simulated gastrointestinal $\mathrm{pH}$ treatment is comparable to the findings of Liyana-Pathirana and Shahidi [17] who also reported an average two- fold increase for the TPC of commercial soft and hard wheat flour upon simulated digestion. D'Archivio et al. [13] and Ortega et al. [18] highlighted that food matrices, interaction of other biomolecules and gut related factors could potentially influence food bioavailability directly or by decreasing polyphenol content in food. A reasonable assumption especially in the context of the current study is that $\mathrm{pH}$ changes, specifically acidification, lead to liberation of phenolics bound to food matrices, potentially enhancing bioavailability by increasing the amount of phenolics available for absorption. This is further supported by Liyana-Pathirana and Shahidi [17] and Baublis et al. [19] who contend that subjection of studied plant samples to gastric $\mathrm{pH}$ treatment increases phenolic compositions and contents. Acidification, as accounted by Kroon et al. [20], is able to free esterified phenolic compounds from fibrous carbohydrates. Furthermore, indicative of the nutritional value of SPF, extraction of phenolics and flavonoids under subjection to $\mathrm{pH}$ adjustment proved to be a good strategy to improve extractability of polyphenols in SPF. SPF treated with gastrointestinal $\mathrm{pH}$ conditions showed a marked increase in extract yield, TPC and TFC, indicating marked stability of SPF-derived bioactive compounds in the gastrointestinal tract. 
Table 2. Total phenolic and flavonoid contents of SPF as affected by simulated gastrointestinal $\mathrm{pH}$ conditions.

\begin{tabular}{ccc}
\hline Sweet Potato Flour (SPF) & $\begin{array}{c}\text { Total Phenolic Content } \\
(\boldsymbol{\mu g} \text { GAE/g SPF) }\end{array}$ & $\begin{array}{c}\text { Total Flavonoid Content } \\
(\boldsymbol{\mu g} \text { RE/g SPF) }\end{array}$ \\
\hline Non-treated & $32.54 \pm 3.17^{\mathrm{a}}$ & $25.64 \pm 4.80^{\mathrm{a}}$ \\
Treated & $76.98 \pm 9.82^{\mathrm{b}}$ & $246.71 \pm 91.65^{\mathrm{b}}$ \\
\hline
\end{tabular}

a,b: Results obtained from means of three determinations \pm standard deviation. Different letters within the same column indicate significant difference $(p<0.05)$.

\subsection{Antioxidant Activity}

Antioxidant activity of SPF, as affected by simulated gastrointestinal $\mathrm{pH}$ conditions, is presented in Figure 1. All five antioxidant assays conducted, concurred that the antioxidant activity of treated SPF was significantly higher $(p<0.05)$ than non-treated SPF. It was found that treated SPF exhibited relatively high 1,1-diphenyl-2-picrylhydrazyl radical (DPPH·) scavenging activity, which is approximately four times the activity shown by non-treated SPF. Correspondingly, 2,2'-azino-bis(3ethylbenzthiazoline-6-sulphonic acid) radical cation (ABTS ${ }^{+}$) scavenging activity and ferric reducing antioxidant power (FRAP) assays also showed that treated SPF displayed higher activities (approximately three times higher) than non-treated sample. Treated SPF was also capable of retarding linoleic acid peroxidation eight times more efficiently than non-treated SPF, as indicated through the use of $\beta$ carotene-linoleate model system. In the iron chelating assay, treated SPF exhibited chelating capacity eight times higher than non-treated SPF.

Figure 1. Antioxidant activity of SPF as affected by simulated gastrointestinal $\mathrm{pH}$ conditions. Results obtained from means of three determinations \pm standard deviation. Trolox was used as a standard in DPPH· scavenging activity, $\mathrm{ABTS} \cdot{ }^{+}$scavenging activity, FRAP and beta-carotene bleaching (BCB) assays. The standard for iron chelating activity assays was ethylenediaminetetra acetic acid (EDTA).

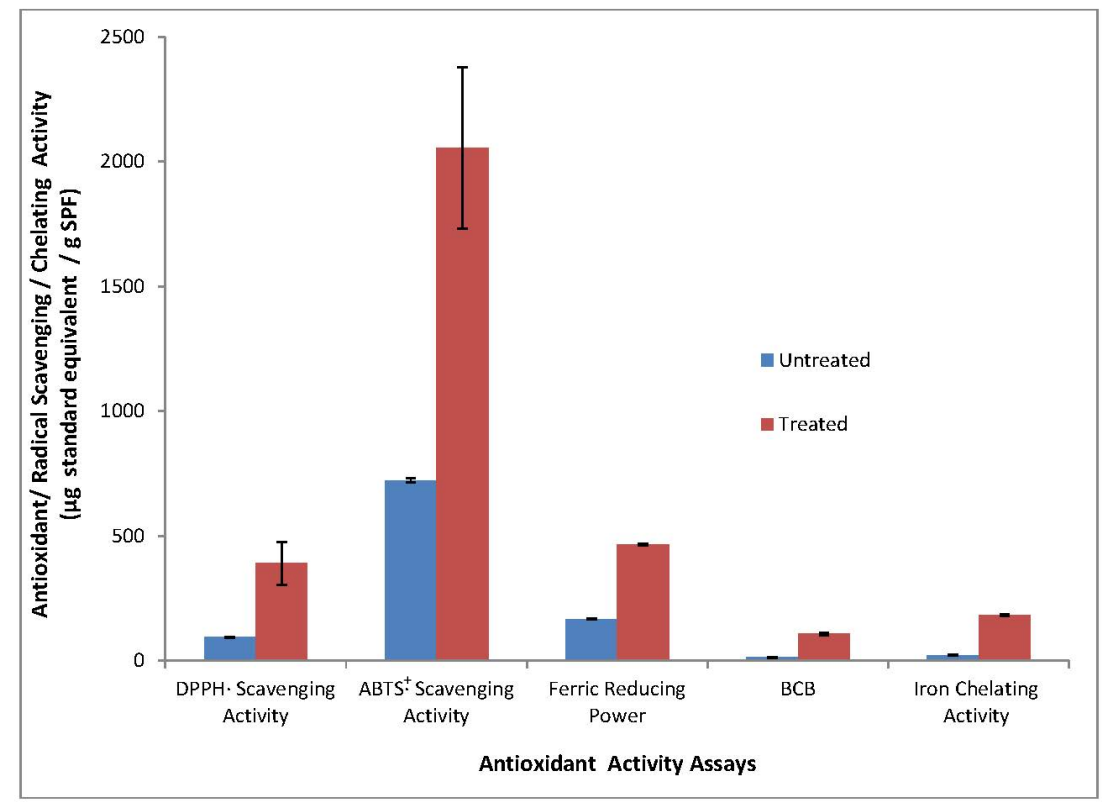


Results from all antioxidant assays indicated that upon digestion of SPF, primary antioxidants, particularly phenolic compounds, might be released from the matrices of SPF and depicted by better radicals scavenging activity and ferric reducing capacity in the treated SPF. Primary antioxidants act to scavenge free radicals and interrupt autoxidation pathways by donating hydrogen atoms or electrons so that free radicals can be converted to a relatively stable form of product. Possessing higher affinities for peroxy radicals than lipids, primary antioxidants react prevalently with peroxy radicals which would eventually retard linoleic acid oxidation. This explains the similar increase in the antioxidant activity exhibited by treated SPF through BCB assay. Interestingly, SPF subjected to gastrointestinal $\mathrm{pH}$ conditions was also found to display stronger secondary antioxidant properties. Under simulated gastrointestinal $\mathrm{pH}$ adjustment, SPF exhibited a higher capability as iron chelator in comparison to its non-treated counterpart. These findings are further supported by the higher TPC and TFC of treated SPF, reported in the former section. Phenolic compounds are important phytochemicals that determine the antioxidant activity in a wide range of botanical samples [21]. Similar improvements in antioxidant capacity were also reported by Baublis et al. [19] whereby gastrointestinal $\mathrm{pH}$ conditions contributes to a marked increase in antioxidant activity of cereals extract as well. Analogously, the antioxidative activity of polyphenols of different sources and wheat flours too, had been reported to be increased after undergoing $\mathrm{pH}$ changes in the gut $[17,22,23]$.

Despite divergent views on the fate of phenolics in gastrointestinal tract, results of antioxidant activity in vivo give a fairly consistent result. Phenolics and other antioxidants consumed orally still produce significant effects to the well-being of the body despite undergoing changes in the gut [12,24]. Specifically in human, pharmacodynamics of phenolics would mostly remain unaffected due to enhanced activity under gut conditions [22]. It had been suggested that changes in chemical structure of phenolics and other compounds could have been responsible for improved antioxidant activity rather than its quantity [16]. Additionally, phenolics potency responsible antioxidant activity is suggested to mainly depend on the degree of deprotonation; therefore it is the degree of hydroxyl radical moiety deprotonation, and not the amount of phenolics present, that causes the antioxidant activity [25]. Going by this hypothesis, the improved antioxidant activity recorded upon treatment may also be attributed to the degree of deprotonation caused by the liberated phenolics upon being subjected to simulated digestion of SPF. Accordingly, consumption of SPF could be promoted as the most likely source of phenolics and antioxidants in human where these pro-health factors appear to be stable when subjected to gastrointestinal $\mathrm{pH}$ changes. Additionally, nutraceutical ingredients with high antioxidant activity have been proven to dampen food deterioration and lipid peroxidation upon incorporation into common dietary products [26]. SPF which exhibited high antioxidant properties especially upon digestion was particularly promising to be utilized as natural food additive to improve food shelf-life stability as well as human nutrition. Nonetheless, it was suggested that acidification could be considered to be incorporated in the extraction of antioxidants from various food and natural products given its capability to produce a dramatic enhancement of antioxidant activity as compared to conventional extraction. 


\section{Experimental Section}

\subsection{Chemicals}

The chemicals used in this study were of analytical reagent or HPLC grade that include: methanol, chloroform and Tween 20 (Fisher Scientific, Loughborough, Leicestershire, UK); linoleic acid, gallic acid, $\beta$-carotene (Type I synthetic, 95\%), sodium bicarbonate, aluminium trichloride $\left(\mathrm{AlCl}_{3}\right)$, potassium dihydrogen phosphate, dipotassium hydrogen phosphate, 6-hydroxy-2,5,7,8-tetramethylchroman2-carboxylic acid (Trolox), 2,2'-azino-bis(3-ethylbenzthiazoline-6-sulphonic acid) (ABTS), potassium persulphate, ferrous chloride $\left(\mathrm{FeCl}_{2}\right)$, ferric chloride $\left(\mathrm{FeCl}_{3}\right)$, potassium ferricyanide $\left[\mathrm{K}_{3} \mathrm{Fe}(\mathrm{CN})_{6}\right]$, ethylenediaminetetra acetic acid (EDTA), 1,1-diphenyl-2-picrylhydrazyl (DPPH), sodium hydroxide $(\mathrm{NaOH})$, Folin-Ciocalteu's phenol reagent and ferrozine (Sigma-Aldrich Co., St. Louis, MO, USA); n-hexane, trichloroacetic acid and hydrochloric acid (HCl) (Merck, Darmstadt, Germany).

\subsection{Samples Preparation}

Sweet potato (Ipomoea batatas) flour was purchased from Sin Guo Co. Pte. Ltd. (Woodlands, Singapore). Two hundred grams of sweet potato flour was mixed with $400 \mathrm{~mL}$ of $\mathrm{n}$-hexane and the mixture was homogenized (Ultra-turax T25 basic, IKA ${ }^{\circledR}$-WERKE GmbH \& Co. KG, Staufen, Germany) for $15 \mathrm{~min}$ at $9500 \mathrm{rpm}$. Subsequently, the mixture was filtered through Whatman No. 2 filter paper and the residues were re-extracted twice following the same procedure. Defatted sweet potato flour were collected and dried in an oven at $50{ }^{\circ} \mathrm{C}$ for $3 \mathrm{~h}$ in order to remove the residual solvent. Finally, defatted flours were passed through a 30 mesh sieve and kept at $-20{ }^{\circ} \mathrm{C}$ prior to the crude phenolics extraction procedure. The finale fat content of sweet potato was $0.73 \pm 0.01 \%$.

\subsection{Crude Phenolics Extraction}

Crude phenolics of defatted flours were extracted according to the procedure described by Baublis et al. [19]. In brief, $10 \mathrm{~g}$ of defatted samples were continuously stirred for $30 \mathrm{~min}$ with $150 \mathrm{~mL}$ of deionised water. The slurries obtained were centrifuged at $4500 \mathrm{rpm}$ for $30 \mathrm{~min}$ at ambient temperature. The resulting supernatants were filtered through Whatman No. 1 filter paper and lyophilised (Virtis Benchtop K Freeze Dryer, SP Industries, Warminster, PA, USA) to obtain the crude phenolics extract. Finally, the yield of crude phenolic extracts was measured before keeping at $-80{ }^{\circ} \mathrm{C}$ for further analyses.

In order to determine the effect of simulated gastrointestinal $\mathrm{pH}$ conditions on phenolic content and antioxidant activity of tested samples, $10 \mathrm{~g}$ of defatted flours were continuously stirred for $30 \mathrm{~min}$ with $150 \mathrm{~mL}$ of deionised water. Then, the mixtures were acidified to $\mathrm{pH} 2$ using $6 \mathrm{~N} \mathrm{HCl}$ and incubated at $37^{\circ} \mathrm{C}$ for $30 \mathrm{~min}$. Subsequently after incubation, the $\mathrm{pH}$ was raised to 7 using $4 \mathrm{~N} \mathrm{NaOH}$ and samples were further incubated for $30 \mathrm{~min}$ at $37{ }^{\circ} \mathrm{C}$. Finally, the treated samples were centrifuged, filtered, lyophilized and stored under the same conditions at which untreated samples were placed. 


\subsection{Total Phenolic and Flavonoid Contents}

Total phenolic content (TPC) of tested samples was determined by Folin-Ciocalteu Reagent assay. In brief, $10 \mathrm{mg}$ of crude phenolic extracts were individually dissolved in $1 \mathrm{~mL}$ of distilled water. Then, $0.1 \mathrm{~mL}$ of these solutions was serially reacted with $0.5 \mathrm{~mL}$ of $10 \%(\mathrm{v} / \mathrm{v})$ Folin-Ciocalteu Reagent and $0.4 \mathrm{~mL}$ of $7.5 \%(\mathrm{w} / \mathrm{v})$ sodium bicarbonate solution. After incubation at $40{ }^{\circ} \mathrm{C}$ for $90 \mathrm{~min}, 200 \mu \mathrm{L}$ of reaction mixtures were loaded into a 96 -well plate. The absorbance of the reaction mixtures was read at $765 \mathrm{~nm}$ using a microplate reader (Opsys MRTM 96-well microplate reader, Dynex Technologies, VA, USA). Gallic acid was used as standard and TPC of tested samples was expressed in microgram gallic acid equivalents ( $\mu \mathrm{g} \mathrm{GAE}) / \mathrm{g}$ SPF.

Total flavonoid content (TFC) of tested samples was determined using a modified method of Meda et al. [27]. Half millilitre of crude phenolic extract solutions was respectively reacted with $2 \%$ (w/v) $\mathrm{AlCl}_{3}$ for $10 \mathrm{~min}$ in a 96-well plate. After that, absorbance of the mixture was measured at $405 \mathrm{~nm}$ using a microplate reader (Opsys MRTM 96-well microplate reader, Dynex Technologies, VA, USA). The standard used in this assay was rutin and TFC of tested samples was expressed in microgram rutin equivalents ( $\mu \mathrm{g} \mathrm{RE}) / \mathrm{g} \mathrm{SPF}$.

\subsection{Antioxidant Activity Assays}

\subsubsection{DPPH Scavenging Activity}

The determination of DPPH radical scavenging activity of tested samples was performed as described previously [4]. In brief, $50 \mu \mathrm{L}$ of crude phenolic extract solutions, with respective concentration, were reacted with $195 \mu \mathrm{L}$ of DPPH methanolic solution $(0.1 \mathrm{mM})$ in a 96 -well plate. Then the mixtures were swirled gently for $1 \mathrm{~min}$ and allowed to stand in dark for $1 \mathrm{~h}$. Finally, absorbance of resulting mixtures was measured using a microplate reader (Opsys MR ${ }^{\mathrm{TM}}$ 96-well microplate reader, Dynex Technologies, VA, USA) at $540 \mathrm{~nm}$. Trolox was used as standard and DPPH radical scavenging activity was expressed as $\mu \mathrm{g}$ Trolox equivalent/g SPF.

\subsubsection{ABTS ${ }^{+}$Scavenging Activity}

ABTS radical cation $\left(\mathrm{ABTS} \cdot{ }^{+}\right.$) scavenging activity of tested samples was determined according to the procedure described by Re et al. [28] with slight modifications. ABTS ${ }^{+}$was produced by reacting $50 \mathrm{~mL}$ of $7 \mathrm{mM} \mathrm{ABTS}^{+}$stock solution with $50 \mathrm{~mL}$ of $2.45 \mathrm{mM}$ potassium persulfate for $24 \mathrm{~h}$ in dark at room temperature. Then, the ABTS. ${ }^{+}$solution was diluted with ethanol to an absorbance of $0.70 \pm 0.02$ at $734 \mathrm{~nm}$ (Pharmaspec uv-1700, Shimadzu, Kyoto, Japan). Subsequently, $950 \mu \mathrm{L}$ of the adjusted solution was reacted with $50 \mu \mathrm{L}$ of crude phenolic extract solutions. The mixtures were vortexed and allowed to react in dark at room temperature for $10 \mathrm{~min}$. Finally, absorbance of the reaction mixtures was read at $734 \mathrm{~nm}$ using a spectrophotometer (Pharmaspec uv-1700, Shimadzu, Kyoto, Japan). Trolox was used as standard and ABTS ${ }^{+}$scavenging activity was expressed as $\mu \mathrm{g}$ Trolox equivalent/g SPF. 


\subsubsection{Ferric Reducing Antioxidant Power (FRAP)}

Ferric reducing antioxidant power of tested samples was measured as described previously [21]. Briefly, $1 \mathrm{~mL}$ of crude phenolic extract solution was respectively mixed with $2.5 \mathrm{~mL}$ of $0.2 \mathrm{M}$ potassium phosphate buffer $(\mathrm{pH} 6.6)$ and $2.5 \mathrm{~mL}$ of $1 \%(\mathrm{w} / \mathrm{v})$ potassium ferricyanide. The mixtures were incubated at $50{ }^{\circ} \mathrm{C}$ for $20 \mathrm{~min}$ followed by addition of $10 \%(\mathrm{w} / \mathrm{v})$ trichloroacetic acid $(2.5 \mathrm{~mL})$ and centrifugation at $3000 \mathrm{rpm}$ for $10 \mathrm{~min}$ at ambient conditions. Finally, $2.5 \mathrm{~mL}$ of supernatants was diluted with equal amount of distilled water and was reacted with $0.5 \mathrm{~mL}$ of $\mathrm{FeCl}_{3}(0.1 \%)$. Finally, absorbance of the reaction mixtures was read at $700 \mathrm{~nm}$ using a spectrophotometer (Pharmaspec uv-1700, Shimadzu, Kyoto, Japan). Trolox was used as standard and FRAP of tested samples was expressed as $\mu \mathrm{g}$ Trolox equivalent/g SPF.

\subsubsection{Beta-Carotene Bleaching Assay (BCB)}

Antioxidant activity of tested samples was evaluated by BCB assay, as described earlier [4]. In brief, $3 \mathrm{~mL}$ of $\beta$-carotene solution ( $1 \mathrm{mg} \beta$-carotene $/ 10 \mathrm{~mL}$ chloroform) were added to $120 \mathrm{mg}$ of linoleic acid and $1200 \mathrm{mg}$ of Tween 20. The mixture was mixed thoroughly and dried under a stream of nitrogen. Immediately, $100 \mathrm{~mL}$ of distilled water were added to the dried mixture to form a $\beta$-carotene-linoleic acid emulsion. In order to determine the antioxidant activity of tested samples, $1.5 \mathrm{~mL}$ of emulsion were added to $20 \mu \mathrm{L}$ of crude phenolic extract solutions followed by incubation, in a water bath, at $50{ }^{\circ} \mathrm{C}$ for $1 \mathrm{~h}$. Finally, absorbance of the reaction mixtures was read at $470 \mathrm{~nm}$ using a spectrophotometer (Pharmaspec UV-1700, Shimadzu, Kyoto, Japan). Trolox was used as standard and antioxidant activity was expressed as $\mu \mathrm{g}$ Trolox equivalent/g SPF.

\subsubsection{Iron Chelating Activity}

Iron chelating activity of tested samples was determined following a method described previously Wang et al. [29]. Initially, $1 \mathrm{~mL}$ of each crude phenolic extract solution was mixed with $50 \mu \mathrm{L}$ of $2 \mathrm{mM} \mathrm{FeCl}_{2}$ followed by addition of ferrozine $(100 \mu \mathrm{L} ; 5 \mathrm{mM})$ in each mixture. The mixtures were thoroughly vortexed and allowed to stand at room temperature for $10 \mathrm{~min}$. After incubation, absorbance of the resulting mixtures was measured at $562 \mathrm{~nm}$ spectrophotometrically (Pharmaspec UV-1700, Shimadzu, Kyoto, Japan). EDTA was used as standard and iron chelating activity was expressed as $\mu \mathrm{g}$ EDTA equivalent/g SPF.

\subsection{Statistical Analysis}

Data was reported as mean \pm standard deviation from triplicate determinations. Analyses of variance (ANOVA) together with LSD and Tukey tests (SPSS for Windows, Version 15; International Business Machines Corp. IBM: New York, NY, USA; 2006) were conducted to identify the significant difference between the samples $(p<0.05)$. 


\section{Conclusion}

The extract yield, total phenolic content (TPC), total flavonoid content (TFC) and antioxidative activity was significantly enhanced when sweet potato flour (SPF) was subjected to simulated gastrointestinal $\mathrm{pH}$ treatment. The $\mathrm{pH}$ adjustment applied to simulate gut digestion could have improved extractability of antioxidants from SPF. Sweet potato is potentially feasible to be developed as functional food ingredients and nutraceutical additives as their active components might be retained and improved in the biological systems.

\section{Acknowledgments}

This project is jointly funded by Universiti Putra Malaysia (Research University Grant Scheme-RUGS; Project No. 04-01-09-0609RU) and Malaysian Ministry of Agriculture and Agro-based Industry (Agri-ScienceFund: Project No. 05-01-04-SF1074). The authors are thankful to Miss Cher-Lynn Soh and Institute of Bioscience, Universiti Putra Malaysia for their constructive technical support and assistance.

\section{References}

1. Halliwell, B. Free radicals, antioxidants, and human disease curiosity, cause, or consequence? Lancet 1994, 344, 721-724.

2. Kris-Etherton, P.M.; Hecker, K.D.; Bonanome, A.; Coval, S.M.; Binkoski, A.E.; Hilpert, K.F.; Griel, A.E.; Etherton, T.D. Bioactive compounds in foods: Their role in the prevention of cardiovascular disease and cancer. Am. J. Med. 2002, 113, 71-88.

3. Ismail, M.; Al-Naqeep, G.; Chan, K.W. Nigella sativa thymoquinone-rich fraction greatly improves plasma antioxidant capacity and expression of antioxidant genes in hypercholesterolemic rats. Free Radic. Biol. Med. 2010, 48, 664-672.

4. Ismail, H.I.; Chan, K.W.; Mariod, A.A.; Ismail, M. Phenolic content and antioxidant activity of cantaloupe (Cucumis melo) methanolic extracts. Food Chem. 2010, 119, 643-647.

5. Iqbal, S.; Younas, U.; Chan, K.W.; Zia-Ul-Haq, M.; Ismail, M. Chemical composition of Artemisia annua L. leaves and antioxidant potential of extracts as a function of extraction solvents. Molecules 2012, 17, 6020-6032.

6. Iqbal, S.; Younas, U.; Sirajuddin; Chan, K.W.; Sarfraz, R.A.; Uddin, M.K. Proximate composition and antioxidant potential of leaves from three varieties of mulberry (Morus spp.): A comparative study. Int. J. Mol. Sci. 2012, 13, 6651-6664.

7. Wanasundara, P.K.J.P.D.; Shahidi, F. Antioxidants: Science, Technology, and Applications. In Bailey's Industrial Oil and Fat Products, 6th ed.; Shahidi, F., Ed.; John Wiley \& Sons, Inc.: Hoboken, NJ, USA, 2005; Volume 1, pp. 431-489.

8. Rice-Evans, C.A.; Miller, N.J.; Paganga, G. Antioxidant properties of phenolic compounds. Trends Plant Sci. 1997, 2, 152-159.

9. Onyeneho, S.N.; Hettiarachchy, N.S. Antioxidant activity, fatty acids and phenolic acids compositions of potato peels. J. Sci. Food Agric. 1993, 62, 345-350. 
10. Rumbaoa, R.G.O.; Cornago, D.F.; Geronimo, I.M. Phenolic content and antioxidant capacity of Philippine sweet potato (Ipomoea batatas) varieties. Food Chem. 2009, 113, 1133-1138.

11. Kunyanga, C.N.; Imungi, J.K.; Okoth, M.W.; Biesalski, H.K.; Vadivel, V. Total phenolic content, antioxidant and antidiabetic properties of methanolic extract of raw and traditionally processed Kenyan indigenous food ingredients. LWT Food Sci. Technol. 2012, 45, 269-276.

12. Chan, K.W.; Khong, N.M.H.; Iqbal, S.; Ismail, M. Simulated gastrointestinal pH condition improves antioxidant properties of wheat and rice flours. Int. J. Mol. Sci. 2012, 13, 7496-7507.

13. D’Archivio, M.; Filesi, C.; Varì, R.; Scazzocchio, B.; Masella, R. Bioavailability of the polyphenols: Status and controversies. Int. J. Mol. Sci. 2010, 11, 1321-1342.

14. Vallejo, F.; Gil-Izquierdo, A.; Perez-Vicente, A.; Garciäa-Viguera, C. In vitro gastrointestinal digestion study of broccoli inflorescence phenolic compounds, glucosinolates, and vitamin $\mathrm{C}$. J. Agric. Food Chem. 2004, 52, 135-138.

15. Perez-Vicente, A.; Gil-Izquierdo, A.; Garciäa-Viguera, C. In vitro gastrointestinal digestion study of pomegranate juice phenolic compounds, anthocyanins, and vitamin C. J. Agric. Food Chem. 2002, 50, 2308-2312.

16. Huang, S.W.; Frankel, E.N.; Schwarz, K.; German, J.B. Effect of pH on antioxidant activity of $\alpha$-tocopherol and trolox in oil-in-water emulsions. J. Agric. Food Chem. 1996, 44, 2496-2502.

17. Liyana-Pathirana, C.M.; Shahidi, F. Antioxidant activity of commercial soft and hard wheat (Triticum aestivum L.) as affected by gastric pH conditions. J. Agric. Food Chem. 2005, 53, 2433-2440.

18. Ortega, N.; Macià, A.; Romero, M.P.; Reguant, J.; Motilva, M.J. Matrix composition effect on the digestibility of carob flour phenols by an in vitro digestion model. Food Chem. 2011, 124, 65-71.

19. Baublis, A.; Decker, E.A.; Clydesdale, F.M. Antioxidant effect of aqueous extracts from wheat based ready-to-eat breakfast cereals. Food Chem. 2000, 68, 1-6.

20. Kroon, P.A.; Faulds, C.B.; Ryden, P.; Robertson, J.A.; Williamson, G. Release of covalently bound ferulic acid from fiber in the human colon. J. Agric. Food Chem. 1997, 45, 661-667.

21. Chan, K.W.; Iqbal, S.; Khong, N.M.H.; Babji, A.S. Preparation of deodorized antioxidant rich extracts from 15 selected spices through optimized aqueous extraction. J. Med. Plants Res. 2011, 5, 6067-6075.

22. Manach, C.; Williamson, G.; Morand, C.; Scalbert, A.; Rémésy, C. Bioavailability and bioefficacy of polyphenols in humans. I. Review of 97 bioavailability studies. Am. J. Clin. Nutr. 2005, 81, 230S-242S.

23. Scalbert, A.; Williamson, G. Dietary intake and bioavailability of polyphenols. J. Nutr. 2000, 130, 2073S-2085S.

24. Frei, B.; Higdon, J.V. Antioxidant activity of tea polyphenols in vivo: Evidence from animal studies. J. Nutr. 2003, 133, 3275S-3284S.

25. Lemańska, K.; Szymusiak, H.; Tyrakowska, B.; 'Nski, R.Z.; Soffers, A.E.M.F.; Rietjens, I.M.C.M. The Influence of $\mathrm{pH}$ on antioxidant properties and the mechanism of antioxidant action of hydroxyflavones. Free Radic. Biol. Med. 2001, 31, 869-881. 
26. Chan, K.W.; Khong, N.M.H.; Iqbal, S.; Ch'ng, S.E.; Babji, A.S. Preparation of clove buds deodorized aqueous extract (CDAE) and evaluation of its potential to improve oxidative stability of chicken meatballs in comparison to synthetic and natural food antioxidants. J. Food Qual. 2012, 35, 190-199.

27. Meda, A.; Lamien, C.E.; Romito, M.; Millogo, J.; Nacoulma, O.G. Determination of the total phenolic, flavonoid and proline contents in Burkina Fasan honey, as well as their radical scavenging activity. Food Chem. 2005, 91, 571-577.

28. Re, R.; Pellegrini, N.; Proteggente, A.; Pannala, A.; Yang, M.; Rice-Evans, C. Antioxidant activity applying an improved ABTS radical cation decolorization assay. Free Radic. Biol. Med. 1999, 26, 1231-1237.

29. Wang, T.; Jónsdóttir, R.; Ólafsdóttir, G. Total phenolic compounds, radical scavenging and metal chelation of extracts from Icelandic seaweeds. Food Chem. 2009, 116, 240-248.

(C) 2012 by the authors; licensee MDPI, Basel, Switzerland. This article is an open access article distributed under the terms and conditions of the Creative Commons Attribution license (http://creativecommons.org/licenses/by/3.0/). 